imparts to the shavings and it is a pretty piece of evidence of how slight and subtle force determines the direction of a spiral. ${ }^{1}$

In the rare specimen in the Cambridge museum of a narwhal with two tusks both the tusks have a sinistral spiral. In the usual one-tusked specimen the left is always developed and also carries througbout a sinistral twist, but the unerupted tusk, 10 inches long, which is buried in the bone, exhibits a perfectly straight fibre and no spiral is to be found. This suggests that the spiral is formed and its direction determined after it leaves the bone and becomes opposed to the waves during growth. The creature ploughs or planes (as does the carpenter) and plays to one side so as to give a sinistral direction to the spiral which develops in growth. When by rare chance a double tusk appears the creature still from habit carries its head to one side and both tusks show the sinistral spiral. The spiral horns of animals such as the koodoo are symmetrically arranged, are right and left by certain rules to which few exceptions ${ }^{2}$ only are found. These horns keep approximately of the same length, partly by being exposed to equal resistances and like conditions during growth. The hoofs of a sheep which had livet among the boggy soil of the Falklands show the necessity of suitable resistances as well as the effect of undue moisture. Each portion of the cloven hoof is of enormous size and length, twisted and intertwined in a complicated twirl.

To return to the question of the length of the lower limbs, it is important to consider the fact that bones increase in length not so much by interstitial deposit as by addition to their ends; that is, by progressive ossification of the layer of cartilage which intervenes between the end of the shaft and the epiphysis Ossification goes on till the component parts of the bone are all united by bony matter, and thus the stature of the individual is determined. If from inflammation or injury an epiphysis be damaged one limb may be shorter than the other, or inflammatory stimulation may even induce an increased length in the bone affected. The skeletons of tortoises, not being subjected to sudden jars, have no epiphyses at the ends of the long bones. Whereas in the leaping frog the extremities of the humerus and femur long remain as separate epiphyses.

The continuous concussions to which the ends of the bones of the lower limbs are exposed, when a vigorous child is excited by its own natural spirit to run about, are doubtless of great value in assisting the growth in length of the lower limbs, which soon lose their infantile character, and become adapted for running and walking. By exposing the lower limbs to the same influences and resistances during their entire growth we manage to maintain them of the same size and of the same length, and gentle jars upon the epiphyses at the joints may be considered favourable to growth.

If, then, by any evil chance one limb be shorter than the other in a growin child, we must find the cause of that shortening, and if there be nothing against the rational plan of treatment-a $a^{f}$ ter study of the epiphyses and joints of the child-it will $n$ it suffice merely to put a boot with a thicker sole on the shorter limb so as to avoid a tilted pelvis and a crooked spine. In such a case carefully planned exercises will be needed, especially directed to the shorter limb, during all the period of growth. The punishment must be maje to fit the crime, and, so soon as the limb can be trained to a sufficient standard of strength, one-legged balancing and hopping exercises will encourage the growth of the weak limb.

It is probable that sometimes when the heel can be brought down in the short leg that there is more shock to the limb on that side during progression until the boot be worn, and if intermittent pressures and succussions are of any value in respect of gaining length this boot may actually retard growth by lessening the shocks, unless suitable exercises be performed with the short limb. No doubt the short limb will require all care to give it rests as well as resistances but the hopping (as at hop-scotch) or one-legged exercises (like the one-legged knee-bender) gradually taken will give a power of poise or balance which is of great advantage.

A child may have quite a strong limb and yet a poor balance, defining balance as the power of maintaining the equipoise under all conditions with the minimum of muscular effort. Continual standing about and long walks are bad for growth and are to be avoided; cycling should only be used

1 "On Spiral Growth," THR LaNGer, Jan. 1st, 1898, p. 24. 2 Nature, Jan. 10th, 1901. to save fatigue in going to or from the playing-field or gymnasium. Long continued cycling is bad and resembles walking on air-cushions which would give no judicious stimulation of the joints. Much cycling for a female child would run some risk of narrowing the pelvis; yet the cycle could be wisely used on the way to the dancing class or school. The natural gait of the child is a hop, skip, and jump, and not the walk two by two of the old-fashioned girls' school.

The difference between the bone-setter and the surgeon is nowhere more apparent than in cases demanding exactly opposite plans of treatment. In cases of disordered joints and epiphyses rest is imperatively demanded, whether in bed or on Thomas's splints, whereas with sound joints and epiphyses the limb must be actively used under supervision of the surgeon. In such a common case as that of a child with one lower limb shorter than the other after an attack of infantile paralysis the nerve-storm has been long past, and it is necessary to remove the effects of the storm on the limb. After tenotomy, passive movements, and massage have been considered or used, then the child with a suitable boot should begin and continue the one-legged exercises which have been chosen as most helpful, and these must be watched and worked hopefully during the time of growth. The exercises chosen must include those which give gentle natural shocks to the joints. The effect on the local circulation of stamping and beating the feet is valuable, and is immediately felt when an Alpine guide beats the half frozen feet of his Herr.

In the foregoing remarks it will be easy to separate the facts from mere speculations. The practical suggestions are founded, I hope, on the facts, and they are set forth in the belief that, with all the care taken to fix and set at rest the joints and limbs, it is easy to neglect stimulation and encouragement of growth when active treatment is needed. The period of growth in the human subject is so prolonged that a surgeon past middle age may not hope to live to see the results of his advice.

Cambridge.

\section{A CASE OF SUBACUTE GLANDERS.}

BY JOHN FAWOETT, M.D., M.R.C.P. LOND., ASSISTANT PHYSICIAN TO GUY'S HOSPITAI;

\section{AND}

WALTER C. C. PAKES, M.R.C.S. ENG., L.R.C.P. LOND. D.P.H. OANTAB., BACTERIOLOGIST TO GUY'S HOSPITAL.

A MAN, aged 45 years, a cab proprietor, was admitted into Gay's Hospital on August 4th, 1900, under the care of one of us. Three weeks before he was taken ill a horse in his stables, supposed to have been suffering from glanders, had been killed. The patient first felt ill on July 9 th, and between this time and the date of admission he had a small abscess on the left forearm, which was opened, and elsewhere on the body several other swellings which did not suppurate. During this period the patient was so ill that he had to remain in bed, his temperature being raised throughout, and on several occasions reaching $104^{\circ} \mathrm{F}$.

On admission the patient, a big stout man, was obviously very ill. He was very tremulous, but breathing comfortably. His temperature was $103.2^{\circ}$, his pulse was 100 , and his respirations were from 30 to 40 per minute. On the left forearm was a shallow sinus which led into the above-mentioned abscess cavity and from which exuded a little thin discharge. Over the back of the right elbow there was some cellulitis and also over the back of the first and second right metacarpal bones. A similar swelling was present on the inner side of the left knee. These swellings scmewhat resembled patches of erythema nodosum No fluctuation was detected in them nor any codema of the surrounding tissue. A few mucous râ!es were heard over the bases of the lungs posteriorly. No physical signs were present on examination of any other of the organs. The urine did not contain albumin. There was no glandular enlargement and rhinorrbce was not present. There was a small furuncle on the buttock, from which a cultivation was made. For the first three days after admission the condition of the patient did not materially alter, but on August 7th be 
became distinctly worse and bad recurrent attacks of diff. after 48 hours its testicles were distinctly enlarged and culty in breathing, saying "he was sure he would be inflamed. It was killed after four days and pus containchoked." On the 8th a crop of small papules, which became ing bacillus mallei was found inside the tunica vaginalis and pustular, appeared on the back. On the 9th there were a few small abscesses in the body of the testicle which on section fresh papules on the right thigh. The left knee-joint was were seen to contain characteristic bacilli. There can be no found to contain some fluid. During this day and the next doubt, therefore, that the case was one of glanders. It was. the attacks of difficulty in breathing became more marked suspected before death that the bacilli which had been found and the patient, becoming gradually worse, died on the were really glanders bacilli, but owing to the contamination

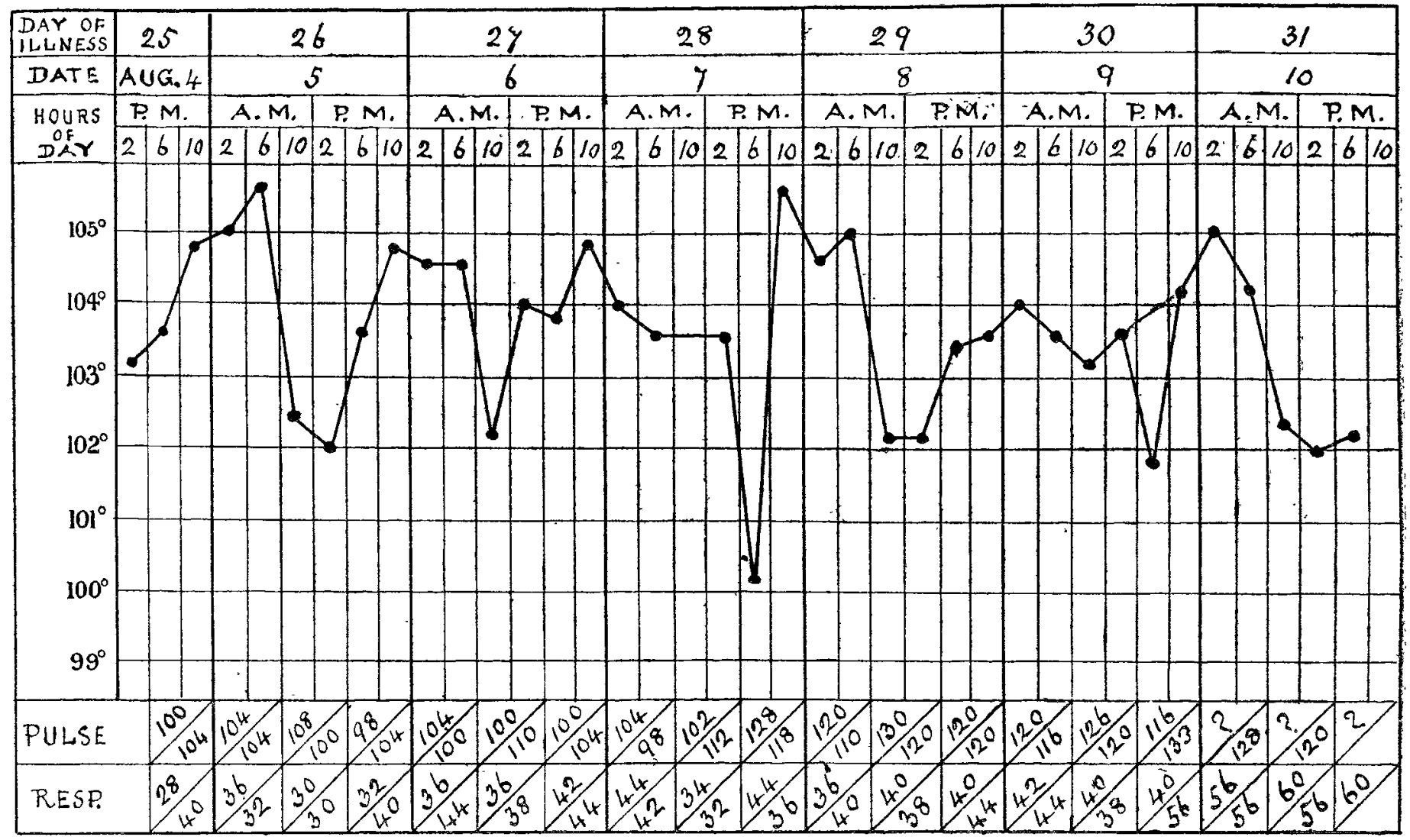

10th. The course of the temperature and pulse is shown on the accompanying chart. Cultivations were made from the furuncles, but up to the time of death no positive result had been obtained.

Necropsy. - The lungs were cedematous, but there were no nodules or any pneumonic areas to be seen. All the other viscera appeared normal to the naked eye and no foci of inflammation or suppuration were present anywhere in, or among, the viscera. The left knee-joint contained a thin, yellowish, glairy fluid and over the right elbow the tissues were infiltrated with a similar oily fluid. Cultivations were made from the heart blood, the spleen, and from the fluid in the region of the elbow and from the left knee-joint.

Remarks. - The case was one of subacute glanders of a month's duration. Daring life, although the history and some of the signs and symptoms suggested the possibility of glanders, yet the clinical evidence, in the absence of bacteriological proof, was not sufficiently clear to allow of a positive diagnosis of glanders being arrived at. Although the patient was very ill the local manifestations of the disease were slight and might have been those of a subacute pyæmia of other origin. They were (1) subcutaneous swellings. (2) a few small furuncles, (3) a small abscess in the left forearm, and (4) the presence of fluid in the left knee-joint. There were no typical ulcers of the skin, no well-marked bullous or pustular eruptions, no rhinorrhœa, and no intra-muscular abscesses, the presence of any of which would have helped to distingush the case with more certainty as one of glanders.

Bacteriology. - The small furuncle upon the buttock was examined microscopically and culturally. Baculli were seen in the pus which resembled the bacillus mallei, but the cultures were full of staphylococci and no bacillus mallei was found upon them. The heart blood, the spleen, and the fluid from the knee-joint were examined and mixed cultures containing a glanders-like bacillus were obtained. These were plated and pure cultures of the bacill us were obtained. The bacillus had all the typical morphological appearances and gave the staining and cultural reactions of the bacillus mallei. A small quantity of a glycerine-agar culture was inoculated into the peritoneum of a male guinea.pig and with other bacteria it was not proved. There would appear to be little doubt that had the abscess upon the forearm been $\in$ xamined bacteriologically the bacillus mallei would have been found. There seems also to be little doubt that had it not been for the bacteriological examination the case would not have been certainly diagnosed as one of glanders.

\section{POSTERIOR BASIC MENINGITIS}

BY HUGH THURSFIELD, M.D. OxON., M.R.C.P. LOND., MEDICAI REGISTRAR AND PATHOLOGIST TO THE HOSPITAL FOR STCK CHILDREN, GREAT ORMOND-STRERT, W.C.

THE disease which is known in this country by the name of "posterior basic meningitis" has always attracted much attention at the Hospital for Sick Children, where the names of Dr. S. J. Gee, Sir Thomas Barlow, Dr. D. B. Lees, and more recently of Dr. Still, are always associated with it. The last-named, while bolding the post of registrar and pathologist, described a diplococcus which be had found constantly present in the meningeal exudations-a diplococcus which in most respects resembles the diplococcus intracellularis of Weichselbanm, the chief differences being the greater vitality of the organism found by Still and the fact that it grew well in broth media, whereas Weichselbaum's diplococcus did not grow at all or very slightly in such media. In the period, July, 1899, to August, 1900, there have been 17 cases of this disease in the medical wards at Great Ormond-street, or at least diagnosed as such, in 12 of which the diagnosis was verified by post-mortem examination. Of the remain. ing five patients two recovered; in two leave to make an examination was refused, and the fifth contracted diphtheria while in the ward and was transferred, owing to pressure upon our available beds, to the fever hospital. These cases, as will be seen later, do not all of them correspond to the 